\title{
The combined effects of soya isoflavones and resistant starch on equol production and trabecular bone loss in ovariectomised mice
}

\author{
Yuko Tousen $^{1}$, Yu Matsumoto ${ }^{1}$, Chiho Matsumoto ${ }^{1,2}$, Yoriko Nishide ${ }^{1,2}$, Yuya Nagahata ${ }^{3}$, \\ Isao Kobayashi ${ }^{3}$ and Yoshiko Ishimi ${ }^{1 *}$ \\ ${ }^{1}$ Department of Food Function and Labeling, National Institute of Health and Nutrition, National Institutes of Biomedical \\ Innovation, Health and Nutrition, 1-23-1 Toyama, Shinjuku-ku, Tokyo 162-8636, Japan \\ ${ }^{2}$ Department of Biotechnology and Life Science, Tokyo University of Agriculture and Technology, 2-24-16 Nakamachi, \\ Koganei, Tokyo 184-8588, Japan \\ ${ }^{3}$ Product Development Laboratory, J-OIL MILLS, Inc., 11 Kagetoricho, Totsuka-ku, Yokohama, Kanagawa 245-O064, Japan \\ (Submitted 30 December 2015 - Final revision received 10 March 2016 - Accepted 17 March 2016)
}

\section{Abstract}

Equol is a metabolite of the soya isoflavone (ISO) daidzein that is produced by intestinal microbiota. Equol has greater oestrogenic activity compared with other ISO, and it prevents bone loss in postmenopausal women. Resistant starch (RS), which has a prebiotic activity and is a dietary fibre, was reported to promote equol production. Conversely, the intestinal microbiota is reported to directly regulate bone health by reducing inflammatory cytokine levels and T-lymphocytes in bone. The present study evaluated the combined effects of diet supplemented with ISO and RS on intestinal microbiota, equol production, bone mineral density (BMD) and inflammatory gene expression in the bone marrow of ovariectomised (OVX) mice. Female ddY strain mice, aged 8 weeks, were either sham-operated (Sham, $n$ 7) or OVX. OVX mice were randomly divided into the following four groups (seven per group): OVX control (OVX); OVX fed 0.05\% ISO diet (OVX + ISO); OVX fed $9 \%$ RS diet (OVX + RS); and OVX fed $0.05 \%$ ISO- and $9 \%$ RS diet (OVX + ISO + RS). After 6 weeks, treatment with the combination of ISO and RS increased equol production, prevented the OVX-induced decline in trabecular BMD in the distal femur by modulating the enteric environment and altered OVX-induced inflammation-related gene expression in the bone marrow. However, there were no significant differences in bone parameters between the ISO + RS and ISO-alone groups in OVX mice. Our findings suggest that the combination of ISO and RS might alter intestinal microbiota and immune status in the bone marrow, resulting in attenuated bone resorption in OVX mice.

\section{Key words: Soya isoflavones: Resistant starch: Bones: Osteoporosis: Intestinal microbiota}

Osteoporosis is a chronic disease characterised by low bone strength, predisposing to an increased risk of fracture ${ }^{(1)}$. Postmenopausal women have a higher risk of developing osteoporosis because of declining oestrogen concentrations associated with menopause ${ }^{(2)}$. One treatment for osteoporosis is hormone replacement therapy; however, its use can result in adverse effects, such as the induction of hormone-dependent breast and uterine cancers ${ }^{(3)}$.

Epidemiological studies indicate that women with high soya intake have a lower risk for osteoporosis compared with those consuming a typical Western diet ${ }^{(4)}$. In addition, many studies indicate that soya isoflavone (ISO)-rich extracts have bone-preserving functions in postmenopausal women ${ }^{(5,6)}$ and oestrogen-deficient animals ${ }^{(7)}$. Soyabean ISO are structurally similar to oestrogen and bind to oestrogen receptors ${ }^{(8)}$, suggesting that they exhibit weak oestrogenic action in various tissues and therefore might prevent postmenopausal disorders such as osteoporosis ${ }^{(6)}$. However, the results of randomised-controlled trials on the bone-protective effects of soya ISO in menopausal women are controversial. Some studies have shown that ISO supplements have a modest effect on bone-sparing or on bone metabolism markers ${ }^{(6,9,10)}$, whereas others considering ISO supplementation with simultaneous Ca and vitamin D supplementation have reported no effects ${ }^{(11-13)}$.

Equol is a metabolite of the ISO daidzein, and it is produced by bacterial microbiota in the distal intestine and colon. It possesses a stronger affinity for oestrogen receptors and induces transcription more strongly compared with other soya ISO ${ }^{(14)}$. Equol has been shown to prevent bone loss in postmenopausal women ${ }^{(15)}$ and oestrogen-deficient animals ${ }^{(16)}$. The clinical effectiveness of soya ISO is proposed to occur following the induction of equol production in the intestine ${ }^{(17)}$. Conversely, Pawlowski et $a l^{(18)}$

Abbreviations: BMD, bone mineral density; CD40L, CD-40-ligand; HAS, high-amylose maize starch; ISO, isoflavone; OVX, ovariectomised mice; RS, resistant starch.

* Corresponding author: Y. Ishimi, fax +81 33205 6549, email ishimi@nih.go.jp 
recently reported that soya ISO are effective bone-preserving agents in postmenopausal women regardless of their equolproducing status. Intestinal bacteria have an essential role in daidzein metabolism, and specific equol-producing bacteria are required ${ }^{(19)}$. Most studies suggest that animals, but not all humans, can produce equol ${ }^{(9,20,21)}$. At least $50-60 \%$ of the Asian population can metabolise daidzein to equol ${ }^{(22)}$, which is remarkably higher than the reported $25-30 \%$ in Western countries ${ }^{(23)}$. The reasons for these differences are unclear, but habitual dietary patterns may influence the metabolism of ISO and the production of equol ${ }^{(24)}$. Lampe et al. ${ }^{(24)}$ reported that diets of equol producers are richer in dietary fibres and carbohydrates compared with those of nonproducers. Therefore, several studies have examined the effect of both prebiotics and probiotics on equol production in an attempt to establish the beneficial effects of ISO $^{(24)}$. Moreover, it is reported that daidzein is metabolised in the colon to equol by bacterial species such as Bifidobacterium spp. and Lactobacillus spp. ${ }^{(25-27)}$. However, the in vivo metabolic processes of these intestinal microbiota remain to be fully elucidated.

Resistant starch (RS) is a type of dietary fibre that includes all starch and starch degradation products that are not absorbed in the small intestine of healthy humans ${ }^{(28)}$. RS is fermented to a large extent by microbiota in the colon, resulting in the production of SCFA, which lowers the $\mathrm{pH}$ in the colon ${ }^{(29)}$. Several studies have characterised the potential of RS to induce alterations in the composition of the gut microbiota and have reported increases in Bifidobacteria, Lactobacillus spp. and Bacteroides ${ }^{(20,30-32)}$.

Oestrogen deficiency results in bone loss associated with altered immune status. The gut microbiota modulates the host metabolism and development of the immune status. Recent studies have suggested an important role for gut-bone signalling pathways and microbiota in regulating bone health via the modification of immune status ${ }^{(33-35)}$. Ohlsson et al. ${ }^{(34)}$ reported that Lactobacillus spp. treatments reduced the expression of inflammatory cytokines in bone, resulting in attenuated bone resorption in ovariectomised (OVX) mice. However, the mechanism by which the gut and the intestinal microbiota regulate bone density is unknown.

We previously reported that equol treatment in OVX mice prevented bone loss and altered bone marrow cell gene expression of the inflammatory indices induced by oestrogen deficiency ${ }^{(36)}$. Therefore, we hypothesised that the intake of a combination of ISO and RS might modulate intestinal microbiota, promote equol production and/or regulate bone inflammation, resulting in cooperative effects on suppressed bone loss caused by oestrogen deficiency. In this study, we examined the combined effects of diet supplemented with ISO and RS on these events using an osteoporotic animal model.

\section{Methods}

\section{Animals and diet}

Female ddY strain mice, aged 8 weeks, were purchased from the Shizuoka Laboratory Animal Center (Shizuoka, Japan). The mice were housed in individual cages in a temperature- and humidity-controlled room $\left(23 \pm 1^{\circ} \mathrm{C}\right.$ and $60 \pm 5 \%$ relative humidity) with a $12 \mathrm{~h}$ light $-12 \mathrm{~h}$ dark cycle. The mice were given free access to an AIN-93G diet with maize oil instead of soyabean oil for $4 \mathrm{~d}$ before surgery was performed ${ }^{(37)}$. All ingredients for the AIN-93G diet were purchased from Oriental Yeast Co., Ltd. The mice were either sham operated (Sham, $n$ 7) or underwent OVX on the same day. OVX mice were randomly divided into four groups ( $n 7$ each): OVX control (OVX); OVX fed $0.05 \%$ ISO-supplemented diet (OVX + ISO); OVX fed $9 \%$ RS-supplemented diet (OVX+RS); and OVX fed $0.05 \%$ ISO- and $9 \%$ RS-supplemented diet (OVX + ISO + RS). The mice were pair-fed their respective diets for $42 \mathrm{~d}$ with free access to distilled water during this period. The ISO and RS concentrations were determined in our previous studies; $0.05 \%$ ISO or $9 \%$ RS diets slightly inhibited bone loss in OVX mice (unpublished results). The sample size calculation was performed before the start of the study, and it indicated that five mice for each group would show a difference of $6.2 \mathrm{mg} / \mathrm{cm}^{2}$ between femur bone mineral density (BMD) means, assuming a SD of $2.6 \mathrm{mg} / \mathrm{cm}^{2}$, with power of 0.80 and a two-sided type 1 error of $0 \cdot 05$. Seven mice in each group were therefore considered an appropriate sample size for the study.

Table 1 shows the composition of the experimental diets, which were prepared according to the AIN-93G formulation ${ }^{(37)}$. Maize oil was used to eliminate any contamination from ISO in soyabean oil. Dry powdered ISO and RS were added to the diet instead of sugar or maize starch, respectively. Supplemented ISO (ISO content: $94.4 \%$, J-OIL MILLS, Inc.) contained the purified ISO conjugates daidzin (55.8\%), glycitin (27.3\%), genistin $(10 \cdot 3 \%)$ and others $(1 \cdot 1 \%)$. Aglycones were present at a concentration of $58.8 \mathrm{mg} / 100 \mathrm{mg}$ of conjugates. The ISO composition was determined by the HPLC method ${ }^{(38)}$. For the supplemented RS, acid-hydrolysed high-amylose maize starch (HAS) was included as $68 \%$ of the dried weight (Amylofiber ${ }^{\circledR}$; J-OIL MILLS, Inc. ${ }^{(39)}$. When preparing the diet, the RS content of acid-hydrolysed HAS was $60 \%$ (wet convention weight). ISO and RS were kindly provided by J-OIL MILLS, Inc.

After $40 \mathrm{~d}$ of treatment, 48 -h urine and faecal samples were collected and stored at $-80^{\circ} \mathrm{C}$ until assayed. Mice were fasted overnight the day before anatomical investigations. After $42 \mathrm{~d}$ of treatment, the mice were euthanised by exsanguination under anaesthesia, weighed and then blood was collected in vacutainers and centrifuged at $7 \boldsymbol{g}$ at $4^{\circ} \mathrm{C}$ for $15 \mathrm{~min}$. The plasma was removed and stored at $-80^{\circ} \mathrm{C}$ until it was assayed. The uterus was also removed and its wet weight was measured. The caecum was removed with its contents, weighed and stored at $-80^{\circ} \mathrm{C}$ until it was assayed. The left femur was also removed to measure BMD, and the right tibia was removed to extract total RNA from the bone marrow cells.

All procedures involving animals were approved by the National Institute of Biomedical Innovation, Health and Nutrition Guidelines for the Care and Use of Laboratory Animals.

\section{Radiographic analysis of the femur}

BMD of the femur was measured by dual-energy X-ray absorptiometry (DEXA, model DCS-6-EX-R; Aloka) and calculated using the bone mineral content of the measured area. The scanned area of the mouse femur was divided into three equal parts: the proximal, midshaft and distal femur. 
Table 1. Composition of the experimental diets $(\mathrm{g} / \mathrm{kg} \text { diet })^{*}$

\begin{tabular}{|c|c|c|c|c|}
\hline Ingredients & Control & ISO & $\mathrm{RS}$ & $\mathrm{ISO}+\mathrm{RS}$ \\
\hline Maize starch & 529.5 & $529 \cdot 5$ & 379.5 & 379.5 \\
\hline Casein & 200 & 200 & 200 & 200 \\
\hline Sucrose & 100 & 99.5 & 100 & 99.5 \\
\hline Maize oil & 70 & 70 & 70 & 70 \\
\hline Cellulose & 50 & 50 & 50 & 50 \\
\hline Mineral mixture* & 35 & 35 & 35 & 35 \\
\hline Vitamin mixture* & 10 & 10 & 10 & 10 \\
\hline L-Cystine & 3 & 3 & 3 & 3 \\
\hline Choline bitartrate & 2.5 & $2 \cdot 5$ & $2 \cdot 5$ & $2 \cdot 5$ \\
\hline Tert-butylhydroquinone & 0.014 & 0.014 & 0.014 & 0.014 \\
\hline Isoflavones† & - & 0.53 & - & 0.53 \\
\hline Acid-hydrolysed high-amylose maize starch & - & - & 150 & 150 \\
\hline
\end{tabular}

Control, control diet; ISO, isoflavones-supplemented diets; RS, resistant starch-supplemented diets; ISO + RS, isoflavone- and resistant starch-supplemented diet.

* Prepared according to the AIN-93G formulation ${ }^{(37)}$.

$\dagger$ The isoflavone conjugates $(94.4 \%$ isoflavones) were daidzin $(55.8 \%)$, glycitin $(27.3 \%)$, genistin $(10.3 \%)$ and others (1.1\%). Aglycones were present at a concentration of $58.8 \mathrm{mg} / 100 \mathrm{mg}$ of conjugates.

$\ddagger 68 \%$ RS was included in acid-hydrolysed high-amylose maize starch (Amylofiber ${ }^{\circledR}$; J-OIL MILLS, Inc.). When preparing the diet, the RS content of high-amylose maize starch was $60 \%$ (the wet convention weight).

\section{Analysis of trabecular microarchitecture by} micro-computed tomography

The distal femurs were scanned at $48-\mu \mathrm{m}$ intervals using a LaTheta experimental animal computed tomography system (Model LaTheta LCT-200; Hitachi Aloka Medical). Distal femur analyses were performed in a region of trabecular bone to the growth plate extending $1.4 \mathrm{~mm}$ towards the diaphysis excluding the outer cortical bone. The trabecular BMD, the ratio of trabecular area, the minimum moment of inertia of cross-sectional areas (MMICA) and the polar moment of inertia of cross-sectional areas (PMICA) were calculated using the LaTheta software (version 1.31; Hitachi Aloka Medical).

\section{Caecal content weight, $\mathrm{pH}$ and $\beta$-glucosidase activity}

Caecal contents were collected, caecal tissue was washed with saline and the weight of the contents was calculated by subtracting the tissue weight from the total weight. The $\mathrm{pH}$ of the caecal contents was measured with a $\mathrm{pH}$ meter (model B-212, Twin Compact pH meter; Horiba). $\beta$-Glucosidase activity was measured by determining the amount of $p$-nitrophenol from $\beta$-pyranoside. The reaction mixture contained $40 \mu \mathrm{l}$ of substrate solution ( $50 \mathrm{~mm}$-phosphate buffer at $\mathrm{pH} 7 \cdot 0$, $1 \mathrm{~mm}$ - $p$-nitrophenyl $\beta$-pyranoside) and $10 \mu \mathrm{l}$ of sample solution at a $1: 9(\mathrm{v} / \mathrm{v})$ dilution of the caecal sample in $50 \mathrm{~mm}$-phosphate buffer at $\mathrm{pH} 7 \cdot 0$. The reaction mixture was incubated for $60 \mathrm{~min}$ at $37^{\circ} \mathrm{C}$, and the $p$-nitrophenol concentration was measured spectrophotometrically at $405 \mathrm{~nm}$ after the addition of $200 \mu \mathrm{l}$ of $0 \cdot 1 \mathrm{M}-\mathrm{NaOH}$. Enzyme activity was expressed as mol of $p$-nitrophenol per whole caecal content in $60 \mathrm{~min}$.

\section{Time-resolved fluoroimmunoassay for urinary daidzein, equol and genistein}

Urinary daidzein, equol and genistein were analysed by the time-resolved fluoroimmunoassay method, as previously reported $^{(40,41)}$. Urine was hydrolysed by glucuronidase and sulphatase. Urinary daidzein, equol and genistein concentrations were determined by fluorescence using a DELFIA Victor
1420 multilabel counter (PerkinElmer). The final results were calculated as follows: concentration $($ read $) \times 1 /$ recovery $\times$ dilution factor $(\mathrm{nmol} / \mathrm{l}) \times$ urine 24 -h volume.

\section{DNA extraction from faeces}

DNA was extracted from the faecal samples according to a method used by Nagashima et al. with modifications ${ }^{(42,43)}$. The faecal samples were suspended in a solution containing 100 mm-TRIS-HCl (pH 9.0) and 40 mм-EDTA after being washed three times with sterile distilled water, and the faeces were then homogenised using a FastPrep FP100A Instrument (MP Biomedicals). DNA was extracted from the suspension using a GC series genomic DNA whole-blood kit and then purified using a Magtration 12GC system (Precision System Science).

\section{$P C R$ conditions and terminal restriction fragment length polymorphism analysis}

Amplification of the faecal 16S ribosomal DNA (16S rDNA), restriction enzyme digestion, size fractionation of terminal restriction fragments and terminal restriction fragment length polymorphism data analysis were performed according to the method used by Nagashima et al. with modifications ${ }^{(42,43)}$. Briefly, PCR was performed using total faecal DNA and primers for 5'-carboxy-fluorescein-labelled 516f and 1510r. The resulting $16 \mathrm{~S}$ rDNA amplicons were treated for $3 \mathrm{~h}$ at $55^{\circ} \mathrm{C}$ with $10 \mathrm{U}$ of $B s / I$ (5'-CCNNNNNINNGG-3') (New England Biolabs). The fluorescentlabelled terminal restriction fragments produced by digestion with $B s / I$ were analysed by electrophoresis on an ABI PRISM 3130xl Genetic Analyzer (Applied Biosystems) in GeneScan mode (injection time was $30 \mathrm{~s}$, and run time was $40 \mathrm{~min}$ ).

\section{RNA extraction from bone marrow of the tibia and quantitative real-time $P C R$}

Total RNA was extracted from the bone marrow of the tibia using Isogen II (Nippon Gene) according to the manufacturer's 
Table 2. Wet weights of the caecal content, $\mathrm{pH}$ and $\beta$-glucosidase activity in mice* (Mean values with their standard errors; $n$ 7)

\begin{tabular}{|c|c|c|c|c|c|c|c|c|c|c|}
\hline & \multicolumn{2}{|c|}{ Sham } & \multicolumn{2}{|c|}{ ovx } & \multicolumn{2}{|c|}{ OVX + ISO } & \multicolumn{2}{|c|}{$O V X+R S$} & \multicolumn{2}{|c|}{$O V X+I S O+R S$} \\
\hline & Mean & SEM & Mean & SEM & Mean & SEM & Mean & SEM & Mean & SEM \\
\hline Wet weight of caecal content $(\mathrm{g})$ & $0.157^{\mathrm{b}}$ & 0.025 & $0.214^{\mathrm{b}}$ & 0.015 & $0.227^{b}$ & 0.022 & $0.474^{\mathrm{a}}$ & 0.037 & $0.494^{a}$ & 0.038 \\
\hline Caecal content $(\mathrm{pH})$ & $7.857^{\mathrm{a}, \mathrm{b}}$ & 0.048 & $8.043^{\mathrm{a}}$ & 0.057 & $8.043^{\mathrm{a}}$ & 0.061 & $7 \cdot 629^{\mathrm{b}, \mathrm{c}}$ & 0.052 & $7.529^{c}$ & 0.078 \\
\hline Caecal $\beta$-glucosidase activity $\dagger$ & $0.777^{\mathrm{b}}$ & 0.123 & $0.653^{\mathrm{b}}$ & 0.145 & $1.209^{b}$ & 0.236 & $1.602^{\mathrm{a}}$ & $0 \cdot 168$ & $1.916^{\mathrm{a}}$ & 0.215 \\
\hline
\end{tabular}

Sham, sham-operated mice fed the control diet; OVX, ovariectomised mice fed the control diet; OVX + ISO, OVX mice fed a $0.05 \%$ isoflavone (ISO)-supplemented diet; OVX + RS, OVX mice fed a $10 \%$ resistant starch (RS)-supplemented diet; OVX + ISO + RS, OVX mice fed a combination of $0.05 \%$ ISO and $10 \%$ RS-supplemented diets.

a,b,c Mean values within a column with unlike superscript letters were significantly different $(P<0.05)$.

* Wet weight of caecal content, caecal content $\mathrm{pH}$ and caecal $\beta$-glucosidase activity were analysed using one-way ANOVA. Differences between groups were assessed by Tukey's post hoc test $(P<0.05)$.

$\dagger$ Mol of $p$-nitro phenol/whole caecal content/60 min.

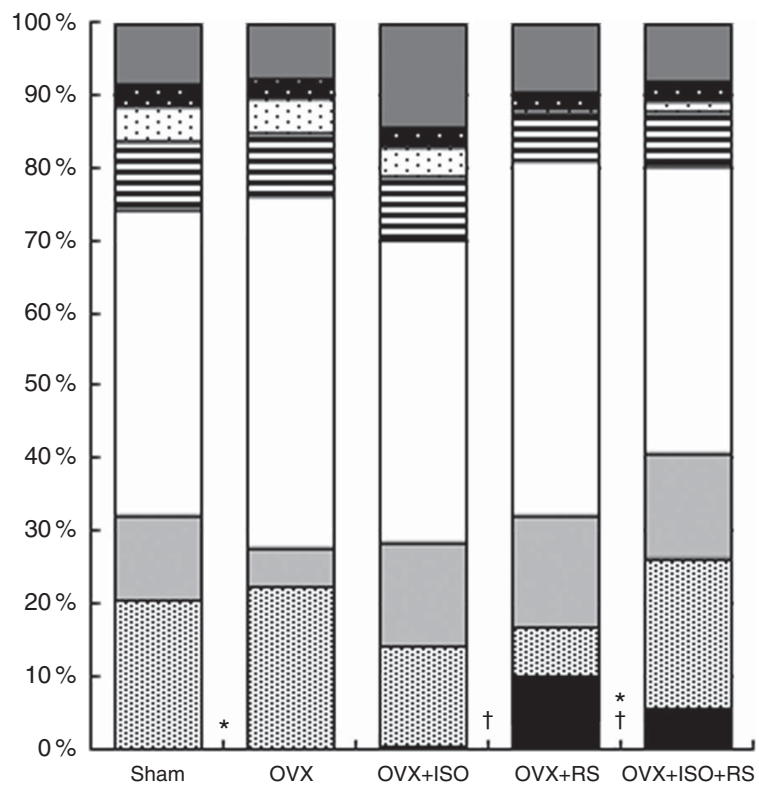

Fig. 1. Composition of caecal intestinal microbiota in Sham mice and ovariectomised (OVX) mice fed either a control diet, an isoflavone (ISO)-supplemented diet (OVX + ISO), a resistant starch (RS)-supplemented diet $(\mathrm{OVX}+\mathrm{RS})$ or a combination of ISO and RS diets $(\mathrm{OVX}+\mathrm{ISO}+\mathrm{RS})$ for $42 \mathrm{~d}$. Values are means $(n 7)$, with their standard errors represented by vertical bars. The influences of ISO and/or RS treatment on the composition of faecal intestinal microbiota were evaluated by ANOVA and Tukey's post hoc test when the data were normally distributed. When the data were not normally distributed and their variances were not equivalent, a non-parametric Kruskal-Wallis test was performed to determine significant differences between groups $(P<0.05)$. ${ }^{*}, \dagger$ Significantly different in the abundance of Bifidobacterium spp. between the Sham and OVX + RS groups, or between the OVX + ISO and OVX + RS groups $(P<0.05)$, respectively. $\square$, Others; $\square$, Clostridium cluster XVIII; $\square$, Clostridium cluster XI; $\square$, Clostridium subcluster XIVa; $\square$, Clostridium cluster IV; $\square$, Prevotella; $\square$, Bacteroides; : $:$, Lactobacillales; $\square$, Bifidobacterium.

instructions. Complementary DNA (cDNA) was synthesised from $1 \mu \mathrm{g}$ of total RNA using PrimeScript RT Master Mix (Takara Bio). cDNA was quantified by real-time PCR using SYBR Premix Ex Taq II (Takara Bio). The PCR conditions were $95^{\circ} \mathrm{C}$ for $30 \mathrm{~s}$, followed by forty cycles of $95^{\circ} \mathrm{C}$ for $5 \mathrm{~s}$ and $60^{\circ} \mathrm{C}$ for $30 \mathrm{~s}$. The primer sequences used in PCR were as follows: $\beta$-actin forward, 5'-CCACAGCTGAGAGGGAAATC-3'; $\beta$-actin reverse, 5 'AAGGAAGGCTGGAAAAGAGC-3'; IL-7R forward, 5'-GCGGACGATCACTCCTTCTG-3'; IL-7R reverse,
5'-AGCCCCACATATTTGAAATTCCA-3'; CD-40-ligand (CD40L) forward, 5'-TCGGGAGCCTTCGAGTCA-3'; CD40L reverse, $5^{\prime}$-GATCCACTGCTGGGCTTCAG-3 ${ }^{\prime(44-46)}$. Results are expressed as the fold-change relative to the controls after normalising to $\beta$-actin gene expression levels.

\section{Statistical analysis}

Data are expressed as mean values with their standard errors. The differences in urinary equol, daidzein and genistein excretions and urinary equol:daidzein ratio between the OVX + ISO and OVX+ ISO + RS groups were examined using the unpaired Student's $t$ test. The significance of differences in BMD, the trabecular area ratio and bone strength parameters of the femur were determined by ANCOVA and Fisher's protected least significant difference test. Body weight was used as a covariate in the analysis of femoral $\mathrm{BMD}$, trabecular BMD, the trabecular area ratio and bone strength parameters of the femur to adjust for possible confounding effects. The influences of ISO and/or RS treatment on the composition of faecal intestinal microbiota were evaluated by ANOVA and Tukey's post hoc test when the data were normally distributed. When the data were not normally distributed and their variances were not equivalent, a non-parametric Kruskal-Wallis test was performed to determine significant differences between groups $(P<0.05)$. The remaining data were analysed using one-way ANOVA. Differences between groups were assessed by Tukey's post hoc test. Differences were considered significant when $P<0 \cdot 05$. Statistical analysis was performed using the SPSS statistics version 19 software (IBM).

\section{Results}

\section{Body and tissue weights}

No statistically significant differences in initial and final body weights or total food intake were observed among groups (data not shown). The uterine weights of mice in the Sham, OVX, $\mathrm{OVX}+\mathrm{ISO}, \mathrm{OVX}+\mathrm{RS}$ and OVX + ISO + RS groups were $84 \cdot 0$ (SEM 12.6), $18 \cdot 0$ (SEM 1.3), 22.3 (SEM 1.2), $17 \cdot 9$ (SEM 0.9) and 23.0 (SEM 4.2) $\mathrm{mg}$, respectively. Uterine weights from all OVX groups were significantly lower than those in the Sham group. Treatment with ISO, RS or a combination of ISO and RS did not affect uterine weight in OVX mice. 
(a)

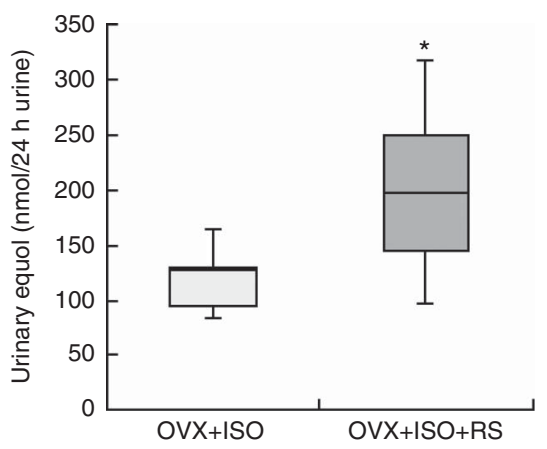

(c)

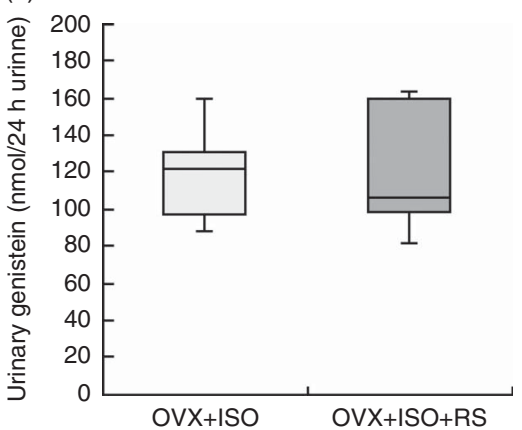

(b)

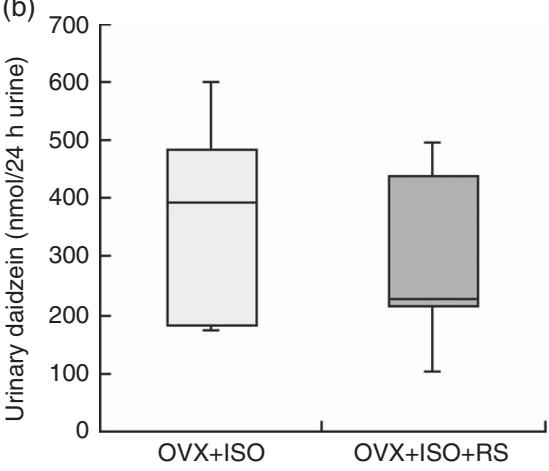

(d)

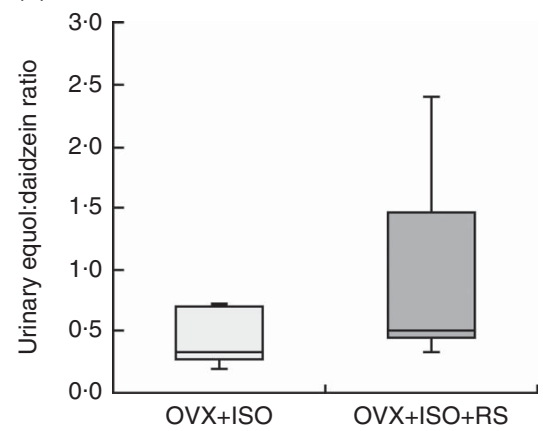

Fig. 2. Urinary excretion of equol, daidzein and genistein, and urinary equol:daidzein ratio in mice fed either an isoflavone (ISO)-supplemented diet (ovariectomised $(\mathrm{OVX})+\mathrm{ISO})$ or a combination of ISO- and resistant starch-supplemented diets $(\mathrm{OVX}+\mathrm{ISO}+\mathrm{RS})$ for $42 \mathrm{~d}$. (a) Urinary equol excretion. (b) Urinary daidzein excretion. (c) Urinary genistein excretion. (d) Urinary equol:daidzein ratio. Values are means $(n 7)$, with their standard errors represented by vertical bars. ${ }^{*}$ Mean values were significantly different between the OVX $+\mathrm{ISO}$ and $\mathrm{OVX}+\mathrm{ISO}+\mathrm{RS}$ groups by unpaired Student's $t$ test $(P<0.05)$.

\section{Caecal content, $\mathrm{pH}$ and $\beta$-g/ucosidase activity}

Table 2 shows the caecal content, $\mathrm{pH}$ and $\beta$-glucosidase activity of OVX and Sham mice. Wet weight of the caecal contents and caecal $\beta$-glucosidase activity were significantly higher in the OVX + RS and OVX + ISO + RS groups compared with the Sham, OVX and OVX + ISO groups. Caecal $\mathrm{pH}$ was significantly lower in the OVX + RS and OVX + ISO + RS groups compared with the OVX and OVX + ISO groups. These results clearly indicated that caecal content, $\mathrm{pH}$ and $\beta$-glucosidase activity were affected by RS intake.

\section{Caecal microbiota analysis}

Human intestinal micro-organisms predominantly consist of the members of approximately nine phylogenetic bacterial groups $^{(42,43)}$. The faecal microbiota was influenced by RS intake (Fig. 1). The ratios of Bifidobacterium spp. in the OVX $+\mathrm{ISO}+\mathrm{RS}$ and OVX+RS groups were 10.29 (SEM 2.46)\% and 5.94 (SEM $2 \cdot 45) \%$, respectively. The abundance of Bifidobacterium spp. was significantly higher in the OVX + RS group $(10 \cdot 27$ (SEM 2.46$) \%$ ) than in the Sham or OVX+ISO groups $(0.27$ (SEM 0.12$) \%$ and 0.62 (SEM 0.34$) \%$, respectively) $(P<0.05)$. However, there were no significant differences in the abundance of Bifidobacterium spp. among the other groups. Furthermore, the abundance of all other bacterial species among all groups was not significantly different.

\section{Urinary excretions of equol, daidzein and genistein}

Urinary excretion of equol, daidzein and genistein was detected in mice treated with ISO, but not in the Sham, OVX and OVX + RS groups (Fig. 2). Urinary equol excretion was significantly higher in the OVX + ISO + RS group compared with the OVX + ISO group (Fig. 2(a)). The product-precursor relationship can be expressed as the excretion ratio of equol:daidzein in urine and is a reliable indicator of the amount of daidzein converted to equol. Although there was no significant difference in the urinary equol:daidzein ratio between the OVX+ISO and OVX+ISO+RS groups, the ratio in the $\mathrm{OVX}+\mathrm{RS}+\mathrm{ISO}$ group tended to be higher than in the OVX+ISO group $(0.705$ (SEM 0.194) and 0.386 (sEm 0.082), respectively) ( $P=0.0654$ ) (Fig. 2(d)). No significant differences were observed in urinary daidzein and genistein excretion between the OVX+ISO and OVX+ISO+RS groups (Fig. 2(b) and (c)).

\section{Bone mineral density, trabecular bone mineral density and} trabecular area ratio of the femur

The BMD of the mice femurs in each group are shown in Fig. 3. The BMD of whole, proximal, middle and distal regions of the femur in the OVX group were significantly lower than those in the Sham group (Fig. 3(A)-(D)). ISO treatment inhibited bone loss in the whole femur and proximal and distal regions of the femur (Fig. 3(A), (B) and (D)). Proximal femur BMD tended to be 

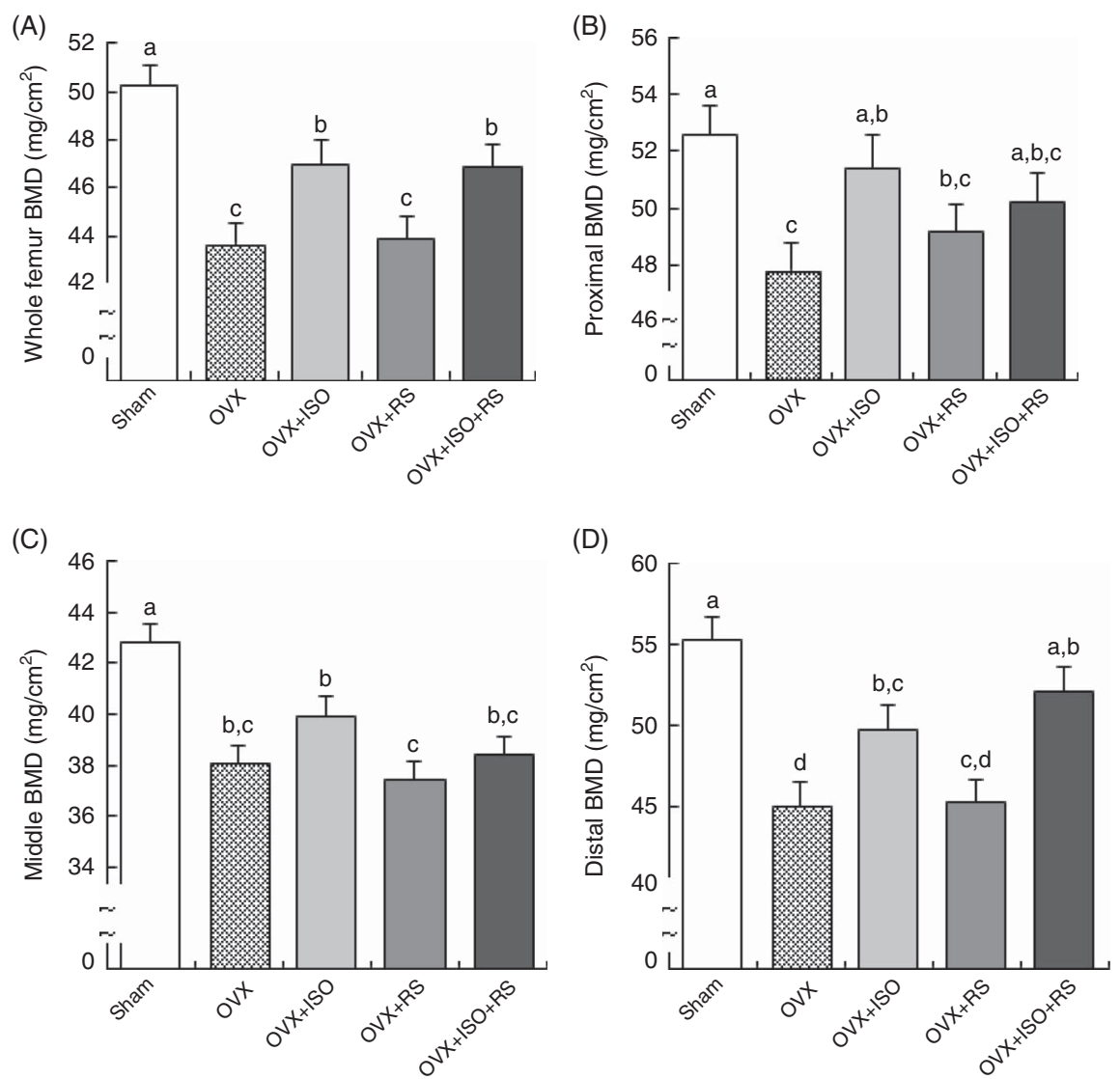

Fig. 3. Bone mineral density of femur was obtained from Sham mice and ovariectomised (OVX) mice fed either a control diet, an isoflavone (ISO)-supplemented diet $(\mathrm{OVX}+\mathrm{ISO})$, a resistant starch (RS)-supplemented diet $(\mathrm{OVX}+\mathrm{RS})$ or a combination of ISO- and RS-supplemented diets $(\mathrm{OVX}+\mathrm{ISO}+\mathrm{RS})$ for $42 \mathrm{~d}$. Bone mineral density (BMD) was measured by dual-energy x-ray absorptiometry analysis. (A) Whole femur BMD. (B) Proximal femur BMD. (C) Middle femur BMD. (D) Distal femur BMD. Values are means $(n 7)$, with their standard errors represented by vertical bars. The significance of differences in BMD was determined by ANCOVA and Fisher's protected least significant difference test. Body weight was used as a covariate in the analysis of femoral BMD to adjust for possible confounding effects. ${ }^{a, b, c, d}$ Mean values with unlike letters were significantly different $(P<0.05)$.

higher in the OVX+RS group than in the OVX group (Fig. 3(B)). Distal femur BMD was slightly higher in the OVX+ISO+RS group than in the OVX+ISO group; however, this difference was not statistically significant (Fig. 3(D)).

To confirm the effect of ISO and RS treatment on distal bone in OVX mice, bone morphometric analysis was performed using micro-computed tomography in the distal femoral metaphysis (Fig. 4 and 5). The connection rods were well maintained in the Sham group (Fig. 4(a)). However, in the OVX group, many of the connecting rods were missing (Fig. 4(b)). Treatment with ISO and ISO+RS prevented trabecular bone loss in OVX mice (Fig. 4(c) and (e)), whereas treatment with RS slightly inhibited trabecular bone loss (Fig. 4(d)). Trabecular BMD and the trabecular area ratio of the distal femur in OVX mice were significantly lower than in Sham mice (Fig. 5(A) and (B)). Conversely, treatment with combined ISO and RS significantly inhibited trabecular bone loss in the distal femur of OVX mice (Fig. 5(A) and (B)). Treatment with ISO alone significantly inhibited the decreased trabecular area ratio. Distal femur MMICA and PMICA bone strength parameters were significantly lower in the OVX group compared with the Sham group (Fig. 5(C) and (D)). However, treatment with combined ISO and RS in OVX mice significantly inhibited the decrease in these parameters in the distal femur (Fig. 5(C) and (D)). On the other hand, these were no statistical differences between the OVX group and the OVX+ISO or the OVX+RS groups.

\section{Expression of inflammation-related genes in bone marrow}

Fig. 6 shows the expression of inflammation-related genes in the bone marrow of the mice. mRNA expression of IL-7R was significantly increased in OVX mice compared with Sham mice, and treatment with ISO and combined ISO + RS significantly reduced IL-7R gene expression (Fig. 6(A)). Moreover, mRNA expression of IL-7R in the OVX + ISO + RS group tended to be lower than that in the OVX+ISO group. Although differences among groups were not significant, expression of the CD40L gene tended to be lower in the OVX + ISO and OVX + ISO + RS groups compared with the other groups (Fig. 6(B)).

\section{Discussion}

In the present study, we examined whether a diet supplemented with ISO and RS could modulate intestinal microbiota, promote 
(a)

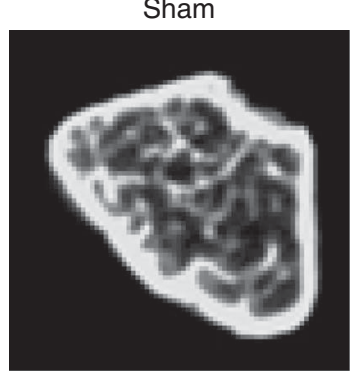

(d)

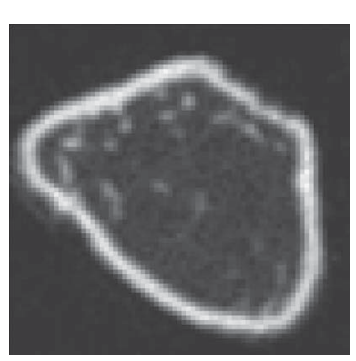

(b)

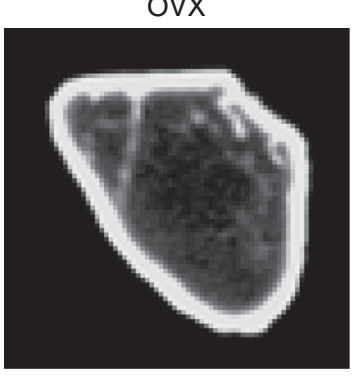

(e)

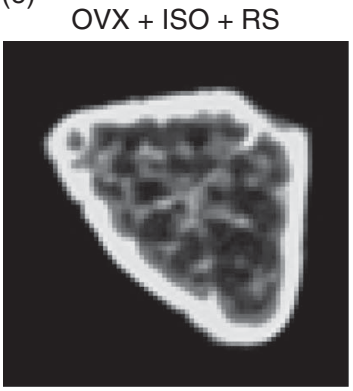

(c)

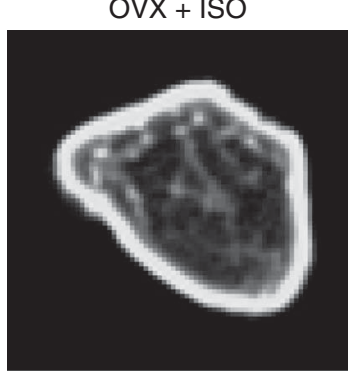

Fig. 4. Micro-computed tomography $(\mu \mathrm{CT})$ scan of trabecular bone of distal femur was obtained from (a) Sham mice and (b) ovariectomised (OVX) mice fed either a control diet, (c) an isoflavone (ISO)-supplemented diet (OVX + ISO), (d) a resistant starch (RS)-supplemented diet (OVX + RS) or (e) a combination of ISO- and RS-supplemented diets (OVX + ISO + RS) for $42 \mathrm{~d}$.

equol production and/or regulate bone inflammation, resulting in the suppression of bone loss caused by oestrogen deficiency in mice. The combination of ISO and RS prevented an ovariectomy-induced decrease in trabecular bone mass and bone strength in the distal femur by modulating the enteric environment, including an increase in the abundance of Bifidobacterium spp. and equol production in the intestine, and regulating inflammation-related genes in the bone marrow.

The ability to produce equol depends on the presence of certain intestinal microbiota, indicating that the enteric environment can affect equol production ${ }^{(47)}$. Our results showed that caecal contents were increased and $\mathrm{pH}$ was decreased in mice fed RS and the combination of ISO and RS compared with the control and ISO-alone diet groups. Moreover, there was an increase in $\beta$-glucosidase activity in the caecal contents and increased equol production in mice fed RS and the combination of ISO and RS. These data are consistent with our previous reports of mice receiving a diet supplemented with a combination of ISO or daidzein and prebiotics, such as fructo-oligosaccharide, polydextrose and raffinose $\mathrm{e}^{(21,48)}$. The activity of $\beta$-glucosidase in the caecal contents is an indicator of the activity of intestinal enzymes that hydrolyse the glycoside bond of ISO conjugates, which stimulates the intestinal absorption of ISO aglycones. These results suggest that RS enhances both the metabolism of ISO conjugates to aglycone and the metabolism of aglycone to equol from daidzein.

Certain Bifidobacterium and Lactobacillus species have been suggested to have an important role in the metabolism of daidzein to equol ${ }^{(25-27,47)}$. The present study demonstrated that the diets containing $0.05 \%$ ISO (containing $55.8 \%$ daidzin as a conjugate) and $9 \%$ RS increased equol production (Fig. 2) by increasing the abundance of Bifidobacterium spp. in the intestinal microbiota (Fig. 1). Previously, we reported that a diet containing $0 \cdot 1 \%$ daidzein and $12 \%$ RS (HAS) promoted equol production and increased the abundance of Bifidobacterium spp. in the faecal microbiota of OVX mice. Categorisation of the types of RS is based on factors that explain their resistance to degradation in the gastrointestinal tract ${ }^{(49)}$, and the functions of RS depend on their resistance to degradation $^{(50,51)}$. In this study, we used hydrolysed-HAS as RS, which is increased in the crystalline regions and which demonstrates increased resistance to enzymatic digestion compared with $\mathrm{HAS}^{(39)}$. Martínez et $a l .{ }^{(50)}$ reported that some types of RS have functional differences in their effects on human faecal microbiota composition, indicating that the chemical structure of RS determines its accessibility by groups of colonic bacteria. Thus, hydrolysed-HAS might be more efficient in metabolising equol from daidzein by stimulating Bifidobacterium spp. in the intestine, resulting in enhanced equol production.

In our study, mice fed a combination of daidzin and RS produced equol, indicating that some rodents are equol producers. However, it might not be easy to enhance equol production in humans, especially in equol non-producers. Some prebiotic supplementations with soya have shown no effect on the equol-producing capacity in humans ${ }^{(52,53)}$. However, further studies are needed to confirm whether RS promotes equol production in humans.

The BMD of the femur, as measured by DEXA, was significantly lower in OVX mice than in Sham mice. ISO alone and the combination of ISO and RS prevented OVX-induced bone loss. Although there was no significant difference 
(A)

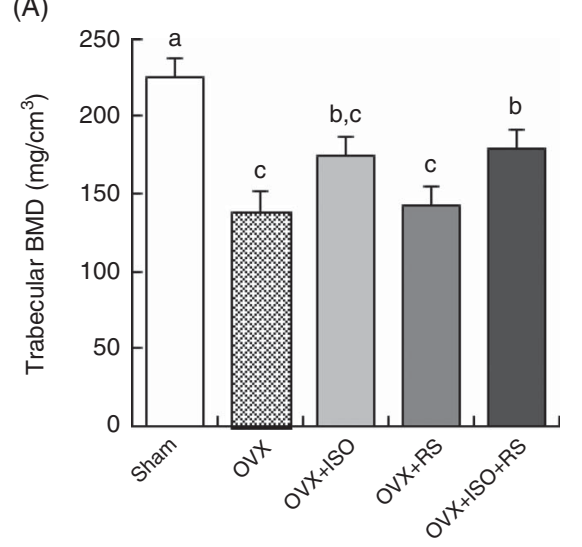

(C)

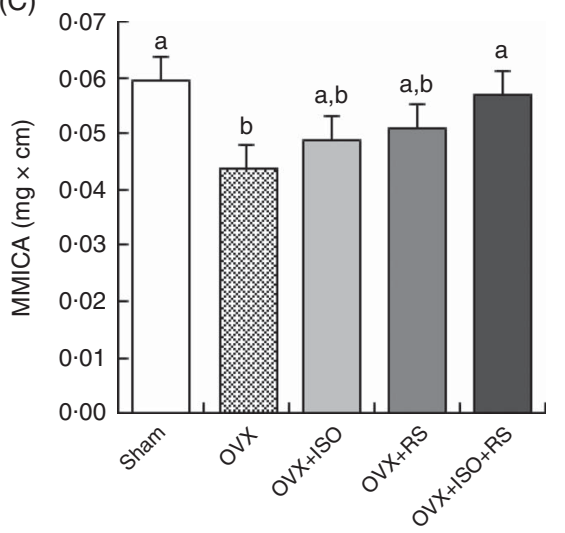

(B)

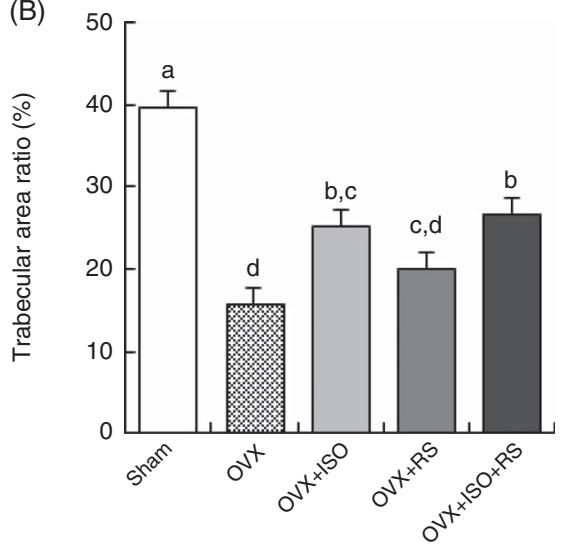

(D)

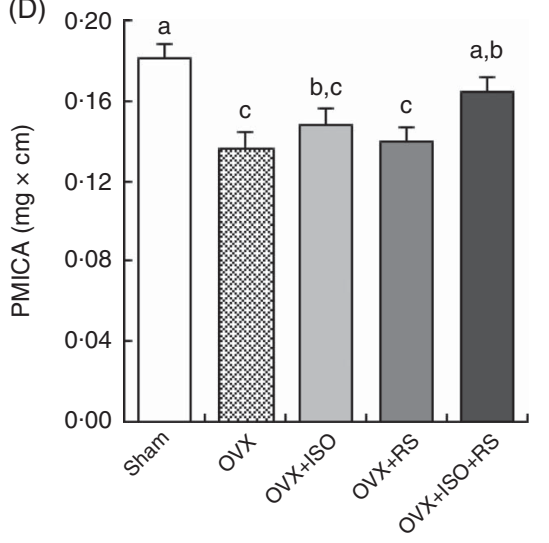

Fig. 5. Trabecular bone mineral density (BMD), the trabecular area ratio and bone strength parameters of the distal femur were obtained from Sham mice and ovariectomised (OVX) mice fed either a control diet, an isoflavone (ISO)-supplemented diet (OVX + ISO), a resistant starch (RS)-supplemented diet (OVX + RS) or a combination of ISO and RS-supplemented diets (OVX + ISO + RS) for $42 \mathrm{~d}$. Trabecular BMD, the trabecular area ratio and bone strength parameters were measured by micro-computed tomography scanning. (A) Trabecular BMD. (B) The trabecular area ratio. (C) Minimum moment of inertia of cross-sectional areas (MMICA). (D) Polar moment of inertia of cross-sectional areas (PMICA). Values are means ( $n 7)$, with their standard errors represented by vertical bars. The significance of differences in trabecular BMD, the trabecular area ratio and bone strength parameters of the femur were determined by ANCOVA and Fisher's protected least significant difference test. Body weight was used as a covariate in the analysis of trabecular BMD, the trabecular area ratio and bone strength parameters of the femur to adjust for possible confounding effects. ${ }^{a, b, c, d}$ Mean values with unlike letters were significantly different $(P<0.05)$.

between the ISO and ISO + RS groups for whole femur BMD, mice fed a combination of ISO and RS maintained their distal BMD more effectively than mice fed ISO alone (Fig. 3(D)). Moreover, trabecular BMD and the trabecular area ratio of the distal femur in the ISO+RS group were significantly higher than those in the OVX group. The specific sites that benefitted most had high amounts of trabecular bone, such as the distal femur. Previously, we reported that equol significantly inhibited the loss of trabecular bone volume in the femoral distal metaphysis, but there was no significant effect in the whole femur ${ }^{(36)}$. This might be because of bone metabolism being faster in trabecular compared with cortical bone. These results indicated that the combination of ISO and RS might be more efficient than ISO alone for trabecular bone BMD in OVX mice. In addition, some part of the ISO effects on trabecular bone might be caused by equol.

Combined ISO and RS treatment significantly inhibited the decrease in bone strength index, including the MMICA and PMICA, in the distal femur of OVX mice (Fig. 5(A) and (B)). Bone quality is recognised to be as important as BMD for bone strength. S-equol is reported to exhibit an ameliorating effect on bone strength ${ }^{(54-56)}$. Mathey et al. ${ }^{(55)}$ reported that bone strength is improved by equol consumption, but not by genistein or daidzein when administered alone. These results suggest that equol is more effective than ISO for the bone strength index in OVX mice.

The association between inflammation and bone loss is well established. Previous studies demonstrated that bone inflammation is increased with oestrogen deficiency and is linked to osteoclastogenesis, and therefore bone resorption is derived by cytokine-producing activated $\mathrm{T}$ cells ${ }^{(57-59)}$. In this study, ISO and/or RS inhibited the increase in OVX-induced IL-7R mRNA expression (Fig. 6(A)). Moreover, although there were no significant differences among all groups, ISO and the combination with RS slightly decreased the expression of CD40L, a key surface ligand expressed on T cells (Fig. 6(B)). IL-7R and CD40L have a key role in bone resorption stimulated by oestrogen deficiency. IL-7 is a pleiotropic immune-regulatory protein predominantly produced by stromal cells and by cells at inflammatory sites. Weitzmann \& Pacifici ${ }^{(60)}$ demonstrated that the administration of IL-7 stimulated 
(A)

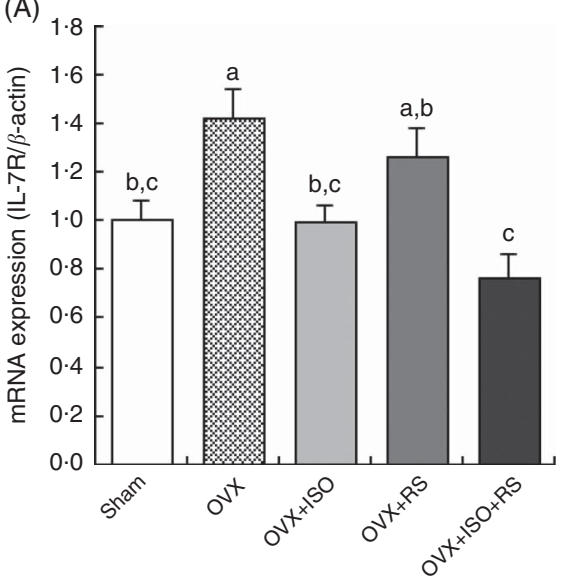

(B)

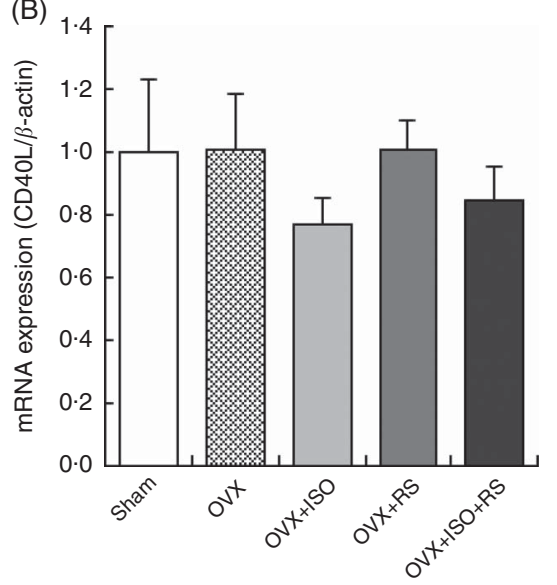

Fig. 6. mRNA expression of bone marrow cells collecting from the tibia was obtained from Sham mice and OVX mice fed either a control diet, an isoflavone (ISO)supplemented diet (OVX + ISO), a resistant starch (RS)-supplemented diet (OVX + RS) or a combination of ISO- and RS-supplemented diets (OVX + ISO + RS) for $42 \mathrm{~d}$. Expression levels of IL-7R and CD-40-ligand (CD4OL) were determined by quantitative real-time PCR. The ordinate axis indicates the relative amount of mRNA compared with sham mice. Gene expression levels were normalised with $\beta$-actin. (A) IL-7R. (B) CD-40-ligand (CD40L). Values are means ( $n$ 7), with their standard errors represented by vertical bars. mRNA expression of bone marrow cells collecting from the tibia was analysed using one-way ANOVA. Differences between groups were assessed by Tukey's post hoc test $(P<0.05)$. ${ }^{\mathrm{a}, \mathrm{b}, \mathrm{c}}$ Mean values with unlike letters were significantly different $(P<0.05)$.

osteoclastogenesis through $\mathrm{T}$ cells. $\mathrm{T}$ cells dysregulate bone homoeostasis in OVX mice through CD40L-mediated cross-talk between themselves and stromal cells, which results in enhanced osteoclastogenesis and osteoblastogenesis ${ }^{(59)}$. Our present results support previous studies in that a diet supplemented with equol for 2 weeks prevented an OVX-induced increase in IL-7R and CD40L in OVX mice ${ }^{(36)}$. These findings suggest that ISO or its combination with RS supplement might alter the inflammation status in bone marrow, resulting in attenuated bone loss in OVX mice.

The consumption of RS changes the composition of the microbiota and promotes the microbial fermentative production of SCFA, which putatively reduce gastrointestinal inflammation. Recent studies suggested an important role for gut-bone signalling pathways and the microbiota in regulating bone health via the modification of immune status ${ }^{(33-35)}$. Britton et al. ${ }^{(35)}$ indicated that one possible indirect mechanism by which probiotic treatment could affect the suppression of bone loss in OVX animals was by alteration of the immune response by changing intestinal microbial communities found in OVX animals. A limitation of the current study is the lack of direct mechanistic experiments assessing the effects between the intestinal microbiota and bone. However, we showed that RS altered the enteric environment, including the caecal $\mathrm{pH}$, weight of caecal content and increased abundance of Bifidobacterium spp. in the intestine, which slightly decreased inflammation-related gene expression in the bone marrow, and slightly suppressed OVX-induced trabecular bone loss. These results raise the possibility that RS affects bone inflammation via SCFA and microbiota in the intestine. Further studies are needed to elucidate these relationships.

In conclusion, treatment with a combination of ISO and RS increased equol production and prevented the OVX-induced decline in trabecular BMD and bone strength parameters in the distal femur by modulating the enteric environment, including an increase in Bifidobacterium spp. and alterations in inflammation-related gene expression in the bone marrow. However, there were no significant differences in bone parameters between the ISO + RS and ISO-alone groups in OVX mice. Our findings suggest that the combination of ISO + RS might alter the microbiota in the intestine and immune status in the bone marrow, resulting in attenuated bone loss in OVX mice. Further studies are necessary to define the mechanism of action of ISO and RS on the intestinal microbiota and immune system and on bone metabolism in oestrogen deficiency.

\section{Acknowledgements}

The authors thank Dr Seiichi Kasaoka of Bunkyo University for his advice on our research.

The present study was supported by J-OIL MILLS, Inc.

The contributions of each author were as follows: Y. I., Y. T., I. K. and Y. N. designed the study; Y. T., Y. M., C. M. and Y. N. carried out the study; Y. T. and Y. M. analysed the data; Y. T. and Y. I. prepared the manuscript.

Y. N. and I. K. are employees of J-OIL MILLS, Inc. All other authors have no conflicts of interest.

\section{References}

1. NIH Consensus Development Panel on Osteoporosis Prevention, Diagnosis and Therapy (2001) Osteoporosis prevention, diagnosis, and therapy. JAMA. 285, 785-795.

2. Masse PG, Dosy J, Tranchant CC, et al. (2004) Dietary macroand micronutrient intakes of nonsupplemented pre- and postmenopausal women with a perspective on menopauseassociated diseases. J Hum Nutr Diet 17, 121-132.

3. Beral V, Bull D, Reeves G, et al. (2005) Endometrial cancer and hormone-replacement therapy in the Million Women Study. Lancet 365, 1543-1551. 
4. Messina MJ (2002) Soy foods and soybean isoflavones and menopausal health. Nutr Clin Care 5, 272-282.

5. Wu J, Oka J, Tabata I, et al. (2006) Effects of isoflavone and exercise on BMD and fat mass in postmenopausal Japanese women: a 1-year randomized placebo-controlled trial. J Bone Miner Res 21, 780-789.

6. Taku K, Melby MK, Takebayashi J, et al. (2010) Effect of soy isoflavone extract supplements on bone mineral density in menopausal women: meta-analysis of randomized controlled trials. Asia Pac J Clin Nutr 19, 33-42.

7. Ishimi Y, Arai N, Wang X, et al. (2000) Difference in effective dosage of genistein on bone and uterus in ovariectomized mice. Biochem Biophys Res Commun 274, 697-701.

8. Schmitt E, Dekant W \& Stopper H (2001) Assaying the estrogenicity of phytoestrogens in cells of different estrogen sensitive tissues. Toxicol In Vitro 15, 433-439.

9. Wu J, Oka J, Ezaki J, et al. (2007) Possible role of equol status in the effects of isoflavone on bone and fat mass in postmenopausal Japanese women: a double-blind, randomized, controlled trial. Menopause 14, 866-874.

10. Marini H, Minutoli L, Polito F, et al. (2007) Effects of the phytoestrogen genistein on bone metabolism in osteopenic postmenopausal women: a randomized trial. Ann Intern Med 146, 839-847.

11. Wong WW, Lewis RD, Steinberg FM, et al. (2009) Soy isoflavone supplementation and bone mineral density in menopausal women: a 2-y multicenter clinical trial. Am J Clin Nutr 90, 1433-1439.

12. Alekel DL, Van Loan MD, Koehler KJ, et al. (2010) The soy isoflavones for reducing bone loss (SIRBL) study: a 3 -y randomized controlled trial in postmenopausal women. Am J Clin Nutr 91, 218-230.

13. Tai TY, Tsai KS, Tu ST, et al. (2012) The effect of soy isoflavone on bone mineral density in postmenopausal Taiwanese women with bone loss: a 2-year randomized double-blind placebo-controlled study. Osteoporos Int $\mathbf{2 3}$, $1571-1580$.

14. Morito K, Hirose T, Kinjo J, et al. (2001) Interaction of phytoestrogens with estrogen receptors alpha and beta. Biol Pharm Bull 24, 351-356.

15. Tousen Y, Ezaki J, Fujii Y, et al. (2011) Natural S-equol decreases bone resorption in postmenopausal, non-equol-producing Japanese women: a pilot randomized, placebo-controlled trial Menopause 18, 563-574.

16. Fujioka M, Uehara M, Wu J, et al. (2004) Equol, a metabolite of daidzein, inhibits bone loss in ovariectomized mice. $J$ Nutr 134, 2623-2627.

17. Lampe JW (2009) Is equol the key to the efficacy of soy foods? Am J Clin Nutr 89, 1664S-1667S.

18. Pawlowski JW, Martin BR, McCabe GP, et al. (2015) Impact of equol-producing capacity and soy-isoflavone profiles of supplements on bone calcium retention in postmenopausal women: a randomized crossover trial. Am J Clin Nutr 102, 695-703

19. Atkinson C, Frankenfeld CL \& Lampe JW (2005) Gut bacterial metabolism of the soy isoflavone daidzein: exploring the relevance to human health. Exp Biol Med (Maywood) 230, $155-170$.

20. Tousen Y, Abe F, Ishida T, et al. (2011) Resistant starch promotes equol production and inhibits tibial bone loss in ovariectomized mice treated with daidzein. Metabolism 60, $1425-1432$.

21. Tousen Y, Uehara M, Kruger MC, et al. (2012) Effects of dietary fibre and tea catechin, ingredients of the Japanese diet, on equol production and bone mineral density in isoflavone-treated ovariectomised mice. J Nutr Sci 1, e13.
22. Akaza H, Miyanaga N, Takashima N, et al. (2004) Comparisons of percent equol producers between prostate cancer patients and controls: case-controlled studies of isoflavones in Japanese, Korean and American residents. Jpn J Clin Oncol 34, 86-89.

23. Setchell KD \& Cole SJ (2006) Method of defining equol-producer status and its frequency among vegetarians. J Nutr 136, 2188-2193.

24. Lampe JW, Karr SC, Hutchins AM, et al. (1998) Urinary equol excretion with a soy challenge: influence of habitual diet. Proc Soc Exp Biol Med 217, 335-339.

25. Xu X, Harris KS, Wang HJ, et al. (1995) Bioavailability of soybean isoflavones depends upon gut microflora in women. J Nutr 125, 2307-2315.

26. Decroos K, Vanhemmens S, Cattoir S, et al. (2005) Isolation and characterisation of an equol-producing mixed microbial culture from a human faecal sample and its activity under gastrointestinal conditions. Arch Microbiol 183, 45-55.

27. Tsangalis D, Ashton J, McGill A, et al. (2002) Enzymic transformation of isoflavone phytoestrogens in soymilk by $\beta$-glucosidase-producing bifidobacteria. J Food Sci $\mathbf{6 7}$, 3104-3113

28. Topping DL, Fukushima M \& Bird AR (2003) Resistant starch as a prebiotic and synbiotic: state of the art. Proc Nutr Soc 62 , 171-176.

29. Topping DL \& Clifton PM (2001) Short-chain fatty acids and human colonic function: roles of resistant starch and nonstarch polysaccharides. Physiol Rev 81, 1031-1064.

30. Zhang Y, Wang Y, Zheng B, et al. (2013) The in vitro effects of retrograded starch (resistant starch type 3 ) from lotus seed starch on the proliferation of Bifidobacterium adolescentis. Food Funct 4, 1609-1616.

31. Conlon MA \& Bird AR (2009) Interactive and individual effects of dietary non-digestible carbohydrates and oils on DNA damage, SCFA and bacteria in the large bowel of rats. $\mathrm{Br} \mathrm{J}$ Nutr 101, 1171-1177.

32. Wang X, Brown IL, Khaled D, et al. (2002) Manipulation of colonic bacteria and volatile fatty acid production by dietary high amylose maize (amylomaize) starch granules. J Appl Microbiol 93, 390-397.

33. Sjogren K, Engdahl C, Henning P, et al. (2012) The gut microbiota regulates bone mass in mice.J Bone Miner Res $\mathbf{2 7}$, $1357-1367$.

34. Ohlsson C, Engdahl C, Fak F, et al. (2014) Probiotics protect mice from ovariectomy-induced cortical bone loss. PLOS ONE 9, e92368.

35. Britton RA, Irwin R, Quach D, et al. (2014) Probiotic L. reuteri treatment prevents bone loss in a menopausal ovariectomized mouse model. I Cell Physiol 229, 1822-1830.

36. Nishide Y, Tadaishi M, Kobori M, et al. (2013) Possible role of S-equol on bone loss via amelioration of inflammatory indices in ovariectomized mice. J Clin Biochem Nutr $\mathbf{5 3}$, 41-48.

37. Reeves PG, Nielsen FH \& Fahey GC Jr. (1993) AIN-93 purified diets for laboratory rodents: final report of the American Institute of Nutrition ad boc writing committee on the reformulation of the AIN-76A rodent diet. J Nutr 123, 1939-1951.

38. Kudou S, Fleury Y, Welti D, et al. (1991) Malonyl isoflavone glycosides in soybean seeds (Glycine max Merrill). Agric Biol Chem 55, 2227-2233.

39. Nagahata Y, Kobayashi I, Goto M, et al. (2013) The formation of resistant starch during acid hydrolysis of high-amylose corn starch. J Appl Glycosci 60, 123-130.

40. Brouwers E, L'Homme R, Al-Maharik N, et al. (2003) Time-resolved fluoroimmunoassay for equol in plasma and urine. J Steroid Biochem Mol Biol 84, 577-588. 
41. Wang GJ, Lapcik O, Hampl R, et al. (2000) Time-resolved fluoroimmunoassay of plasma daidzein and genistein. Steroids 65, 339-348.

42. Nagashima K, Mochizuki J, Hisada T, et al. (2006) Phylogenetic analysis of $16 \mathrm{~S}$ ribosomal RNA gene sequences from human fecal microbiota and improved utility of terminal restriction fragment length polymorphism profiling. Biosci Microflora 25, 99-107.

43. Nagashima K, Hisada T, Sato M, et al. (2003) Application of new primer-enzyme combinations to terminal restriction fragment length polymorphism profiling of bacterial populations in human feces. Appl Environ Microbiol 69, 1251-1262.

44. Kong CS, Kim JA, Eom TK, et al. (2010) Phosphorylated glucosamine inhibits adipogenesis in 3T3-L1 adipocytes. J Nutr Biochem 21, 438-443.

45. Grenningloh R, Tai TS, Frahm N, et al. (2011) Ets-1 maintains IL-7 receptor expression in peripheral T cells. J Immunol 186, 969-976.

46. Schroder K, Vecchione C, Jung O, et al. (2006) Xanthine oxidase inhibitor tungsten prevents the development of atherosclerosis in ApoE knockout mice fed a Western-type diet. Free Radic Biol Med 41, 1353-1360.

47. Yuan JP, Wang JH \& Liu X (2007) Metabolism of dietary soy isoflavones to equol by human intestinal microflora-implications for health. Mol Nutr Food Res 51, 765-781.

48. Ohta A, Uehara M, Sakai K, et al. (2002) A combination of dietary fructooligosaccharides and isoflavone conjugates increases femoral bone mineral density and equol production in ovariectomized mice. J Nutr 132, 2048-2054.

49. Englyst HN, Kingman SM \& Cummings JH (1992) Classification and measurement of nutritionally important starch fractions. EurJ Clin Nutr 46, Suppl. 2, S33-S50.

50. Martínez I, Kim J, Duffy PR, et al. (2010) Resistant starches types 2 and 4 have differential effects on the composition of the fecal microbiota in human subjects. PLOS ONE 5, e15046.
51. Jacobasch G, Dongowski G, Schmiedl D, et al. (2006) Hydrothermal treatment of Novelose 330 results in high yield of resistant starch type 3 with beneficial prebiotic properties and decreased secondary bile acid formation in rats. BrJ Nutr 95, 1063-1074.

52. Tousen Y, Uehara M, Abe F, et al. (2013) Effects of short-term fructooligosaccharide intake on equol production in Japanese postmenopausal women consuming soy isoflavone supplements: a pilot study. Nutr J 12, 127.

53. Larkin TA, Price WE \& Astheimer LB (2007) Increased probiotic yogurt or resistant starch intake does not affect isoflavone bioavailability in subjects consuming a high soy diet. Nutrition $\mathbf{2 3}$, 709-718.

54. Kimira Y, Katsumata S, Suzuki K, et al. (2012) Comparative activities of the S-enantiomer and racemic forms of equol on bone fragility in ovariectomized mice. Biosci Biotechnol Biochem 76, 1018-1021.

55. Mathey J, Mardon J, Fokialakis N, et al. (2007) Modulation of soy isoflavones bioavailability and subsequent effects on bone health in ovariectomized rats: the case for equol. Osteoporos Int 18, 671-679.

56. Sehmisch S, Erren M, Kolios L, et al. (2010) Effects of isoflavones equol and genistein on bone quality in a rat osteopenia model. Phytother Res 24, Suppl. 2, S168-S174.

57. Kong YY, Feige U, Sarosi I, et al. (1999) Activated T cells regulate bone loss and joint destruction in adjuvant arthritis through osteoprotegerin ligand. Nature 402, 304-309.

58. Clowes JA, Riggs BL \& Khosla S (2005) The role of the immune system in the pathophysiology of osteoporosis. Immunol Rev 208, 207-227.

59. Li JY, Tawfeek H, Bedi B, et al. (2011) Ovariectomy disregulates osteoblast and osteoclast formation through the T-cell receptor CD40 ligand. Proc Natl Acad Sci US A 108, 768-773.

60. Weitzmann MN \& Pacifici R (2006) Estrogen regulation of immune cell bone interactions. Ann N Y Acad Sci 1068, 256-274. 